\title{
NOUVELLE
}

\section{Les protéines Piezo forment une nouvelle famille de canaux ioniques activés mécaniquement}

CRN2M-UMR 7286, Université Aix-Marseille, Faculté de médecine, Secteur nord, 13015 Marseille, France. coste@scripps.edu
> On désigne par mécanotransduction la conversion des forces mécaniques en signaux biologiques. La mécanotransduction nous permet de détecter différentes sortes de stimulations mécaniques telles que les caresses, les coups violents ou les vibrations sonores impliquées dans l'audition, et de les convertir en signaux électriques. La mécanotransduction n'est cependant pas limitée aux systèmes sensoriels puisqu'elle est impliquée dans de nombreux autres processus biologiques fondamentaux, tels que la régulation de la pression artérielle, la détection du flux rénal et l'homéostasie musculaire ou osseuse.

En utilisant des préparations ex vivo de cellules ciliées auditives de grenouille, Corey et Hudspeth [1] ont montré à la fin des années 1970 que le temps de latence entre la stimulation mécanique et la réponse électrique excluait la participation de seconds messagers. Ils ont postulé l'existence de canaux ioniques activés directement par les forces mécaniques [1]. Ce postulat fut validé quelques années plus tard par les premiers enregistrements de canaux activés mécaniquement [2]. Bien que certains canaux ioniques activés mécaniquement aient été identifiés et caractérisés, notamment chez les invertébrés, l'identification de tels canaux ioniques chez les mammifères reste l'une des questions les plus importantes dans le domaine de la transduction sensorielle [3].

\section{Identification des protéines Piezo}

Les protéines Piezo sont impliquées dans la mécanotransduction et ont été iden- tifiées par criblage des cellules Neuro2A (une lignée cellulaire issue de neuroblastes de souris) à l'aide de petits ARN interférents (siRNA) [4, 5]. Les protéines Piezo sont nécessaires et suffisantes à l'induction de courants activés mécaniquement. Elles sont composées de plus de 2000 acides aminés et possèdent entre 30 et 40 domaines transmembranaires. Elles n'ont aucune homologie avec d'autres protéines ou canaux ioniques connus à ce jour. Si cette étude initiale $[4,5]$ montre que les protéines Piezo sont impliquées fonctionnellement dans la formation de canaux ioniques activés mécaniquement, elle n'a cependant pas permis d'établir précisément leur nature moléculaire. Ces protéines constituent-elles une nouvelle famille de canaux ioniques, ou confèrent-elles la mécanosensibilité à des canaux ioniques encore non identifiés?

\section{Les protéines Piezol forment} des homotétramères sans protéines associées

En utilisant des approches complémentaires, nous avons étudié la stœchiométrie des protéines Piezo, ainsi que leur association avec d'autres protéines [6]. En utilisant une construction de la protéine Piezol fusionnée avec de la GFP (green fluorescent protein) et exprimée dans des ovocytes de xénope, nous avons montré, par des expériences d'imagerie sur cellules vivantes, qu'au moins quatre molécules de GFP (et donc de Piezol) étaient détectées par complexe. Ces expériences suggèrent donc que les pro- téines Piezo s'associent pour former des homotétramères.

Afin de tester si les protéines Piezo sont associées à d'autres protéines, et notamment à des canaux ioniques, nous avons analysé biochimiquement les complexes Piezol purifiés à partir de cellules HEK293T surexprimant une construction Piezol fusionnée avec la GST (glutathion $S$-transferase). Aucune autre protéine n'a été détectée par spectrométrie de masse dans ces complexes. De plus, des expériences de migration sur gel natif montrent que ces mêmes complexes ont un poids moléculaire d'environ 1,2 millions de dalton, ce qui correspond au poids combiné de quatre molécules de Piezol. Enfin, le prétraitement des cellules exprimant Piezol par des agents de couplage covalent induit l'apparition de complexes de haut poids moléculaire identifiables sur des gels dénaturants et qui correspondent à des homomultimères de Piezol. L'ensemble de ces résultats suggère que les protéines Piezol forment des homotétramères sans protéines associées.

\section{Les protéines Piezol forment}

\section{des canaux ioniques}

L'incorporation de la protéine Piezol au sein de membranes artificielles a permis de tester si Piezol formait des canaux ioniques per se. Les protéines Piezol purifiées et insérées dans deux types différents de bicouches lipidiques induisent des canaux ioniques avec des propriétés similaires à celles induites par l'expression de Piezol dans des lignées cellulaires : une perméabilité cationique 
non sélective et un bloc du pore de conduction par le rouge de ruthénium [6]. Ces résultats révèlent que les protéines Piezo constituent une nouvelle famille de canaux ioniques.

Les protéines Piezo sont impliquées dans la sensibilité mécanique douloureuse

Chez les eucaryotes, beaucoup d'animaux et de plantes ne possèdent qu'un seul gène Piezo, alors que deux gènes sont présents chez les vertébrés. Chez la souris, Piezol et 2 sont exprimées dans de nombreux tissus mécanosensibles. Dans les neurones sensoriels périphériques impliqués dans le toucher et la douleur, Piezo2 contribue aux courants activés mécaniquement à adaptation rapide, un des trois principaux types de courants caractérisés dans ces neurones [5]. Ces données suggèrent que Piezo2 pourrait être impliquée dans certaines modalités du système somatosensoriel. Nous avons testé le rôle physiologique des protéines Piezo chez la drosophile. Chez cet animal comme chez tous les insectes, un seul gène Piezo est présent et son expression hétérologue induit un courant activé mécaniquement [6]. L'inactivation du gène Piezo spécifiquement dans les neurones nociceptifs de la larve de drosophile diminue les réponses aux stimulations mécaniques doulou- reuses, sans affecter les réponses aux stimulations thermiques [7]. Un courant activé mécaniquement dépendant de l'expression de Piezo est détecté dans ces neurones. De plus, la réexpression de la protéine Piezo spécifiquement dans les neurones de larves knock-out pour le gène Piezo restaure le phénotype. Ces résultats indiquent que Piezo est impliquée dans la sensibilité mécanique douloureuse chez la drosophile au stade larvaire, et ils constituent la première démonstration de l'importance physiologique des gènes Piezo in vivo.

\section{Conclusion}

Ces travaux montrent que les protéines Piezo forment une nouvelle classe de canaux ioniques impliqués dans la mécanotransduction, et notamment dans la sensibilité mécanique douloureuse chez la drosophile. Les patrons d'expression de Piezol et 2 sont étendus à de nombreux tissus, ce qui suggère leur implication dans de nombreux processus biologiques chez les mammifères. Deux études récentes ont ainsi mis en évidence le rôle de Piezol dans le contrôle du nombre de cellules épithéliales [8] et dans la régulation du volume érythrocytaire [9]. L'étude approfondie des protéines Piezo et de leurs fonctions devrait permettre de mieux comprendre la mécanotransduction à des niveaux moléculaires et cellulaires, mais également à des niveaux plus intégrés, et ce dans divers processus biologiques. $\diamond$ Piezo proteins form a new class of mechanically activated ion channels

\section{REMERCIEMENTS}

L'auteur tient à remercier le Dr N. Grillet pour ses commentaires pertinents sur le manuscrit.

\section{LIENS D'INTÉR̂̂T}

L'auteur déclare n'avoir aucun lien d'intérêt concernant les données publiées dans cet article.

\section{RéFÉRENCES}

1. Corey DP, Hudspeth AJ. Response latency of vertebrate hair cells. Biophys J $1979 ; 26: 499-506$.

2. Guharay F, Sachs F. Stretch-activated single ion channel currents in tissue-cultured embryonic chick skeletal muscle. J Physiol 1984 ; 352 : 685-701.

3. Chalfie M. Neurosensory mechanotransduction. Nat Rev Mol Cell Biol 2009; 10 : 44-52.

4. Coste B. Détecter la pression? Identification de deux protéines activées par les forces mécaniques. Med Sci (Paris) $2011 ; 27: 17-9$.

5. Coste B, Mathur J, Schmidt M, et al. Piezol and Piezo2 are essential components of distinct mechanically activated cation channels. Science 2010 ; 330 : 55-60.

6. Coste B, Xiao B, Santos JS, et al. Piezo proteins are pore-forming subunits of mechanically activated channels. Nature 2012 ; 483 : 176-81.

7. Kim SE, Coste B, Chadha A, et al. The role of Drosophila Piezo in mechanical nociception. Nature 2012 ; 483 : 209-12.

8. Eisenhoffer GT, Loftus PD, Yoshigi M, et al. Crowding induces live cell extrusion to maintain homeostatic cell numbers in epithelia. Nature 2012 ; 484 : 546-9.

9. Zarychanski R, Schulz VP, Houston BL, et al. Mutations in the mechanotransduction protein PIEZOl are associated with hereditary xerocytosis. Blood 2012 ; 120 : 1908-15.

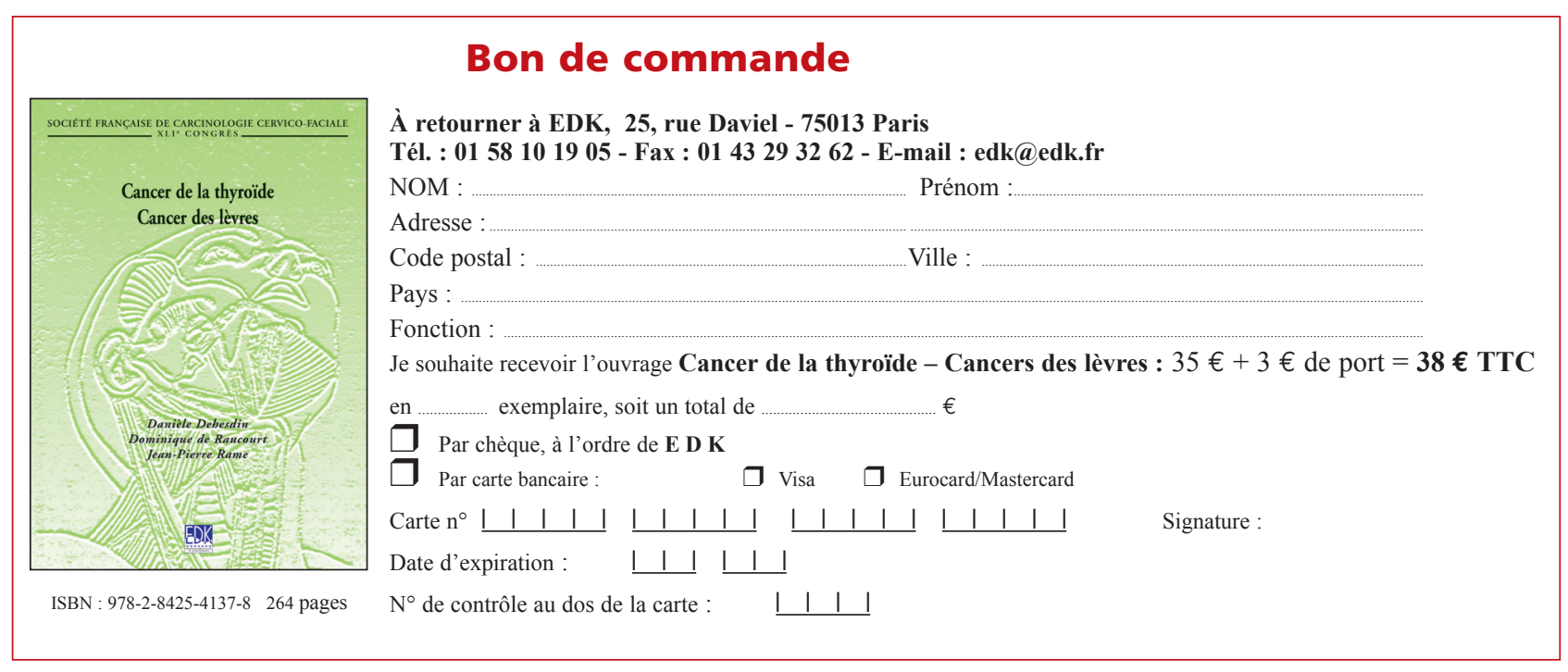

